\title{
Weird Science: Challenging EBSD
}

\author{
C. Necker, R. Forsyth, P. Papin, E. Luther \\ Los Alamos National Laboratory, MST-6: Metallurgy, MS G770, Los Alamos, NM 87544
}

There are three critical and challenging stages in the art of EBSD: sample preparation, data collection, and data analysis. Using metals and alloys as a backdrop, several examples will be used to illustrate techniques for overcoming challenges in all three areas. Without high quality sample preparation you have no patterns and thus no data. Flexibility and the willingness to try alternative prep techniques should allow you to follow the credo "if it is crystalline, I will get patterns". One example of how different preparation makes in pattern quality is the preparation of sintered uranium oxide. Typical preparation includes polishing through $1 \mu \mathrm{m}$ and $0.25 \mu \mathrm{m}$ diamond pastes. The resulting patterns were less than stellar (Figure 1.) A short duration polish on colloidal silica produced outstanding patterns. Sharp patterns were needed to help differentiate matrix material from voids in these less than fully dense samples.

Half of the challenge of data collection is to understand what the customer wants. Without this knowledge you are wasting beam time. The other half of the challenge is to understand how to manipulate the data collection variables to grab the appropriate data. The less than fully dense situation with the uranium oxide also presents a data collection issue due to the $3 \%-6 \%$ void volume. Typical software settings for data collection are normally optimized to seek patterns whenever possible. Even the slightest hint of intensity on the camera is interpreted as a pattern. This condition is problematic in materials with voids or pores. When the beam strikes the edge of voids or falls into the voids non-band artificial lighting conditions persist and are interpreted as patterns, resulting in artificial microstructure features. The software we use (EDAX/TSL) has numerous Hough parameter features that can be adjusted to ensure that the beam interaction with the voids results in a 'no solution' condition rather than the creation of an artificial feature. This is done by characterizing the number and intensity of bands typical of beam interactions with the matrix, void edges and void bodies. Void edges produce lower intensity patterns than the matrix. Void bodies produce lower intensity patterns and fewer patterns than the matrix. The sharp, strong matrix patterns facilitate differentiation from the void edges and bodies by using appropriate maximum peak number and minimum peak magnitude Hough cut-offs.

After successfully prepping the sample and garnering data, there is the challenge of analyzing the data to provide relevant results without introducing artificial features or results. Like most powerful image analysis tools, a myriad of results are possible from any one data set. In the case of the analysis of partially dense uranium oxide the challenge was to properly evaluate the volume fraction of voids and grain size. Various clean-up routines and data partitioning algorithms were used to delineate the voids from the grains and, at the same time, meld single and double point artificial features into the structure. This effect of properly massaging the data is seen in Figure 2. In some cases, as with the forensic restoration of serial numbers, the analytical challenge was to determine which analytical techniques were best suited to highlight defaced serial numbers. In such cases, it is helpful to think about the metallurgical aspects before performing the analyses because knowledge of prior processing often will help one develop analytical choices. In this case, the knowledge that the serial numbers were die stamped 
suggested that we may have strained material with the expectation of low pattern quality, potential changes in texture, localized misorientations and fragmented grains. Figure 3 shows the crystal direction map and the image/pattern quality map for one number in a serial number series. It is pretty clear that the die stamping operation has not imposed a significant enough change in crystallographic orientation to be seen clearly. However, the localize strain is very clearly evident in the image quality map.

Through careful experimental design and knowledge of prior processing, these challenges can be overcome allowing us to further challenge the capabilities of EBSD.
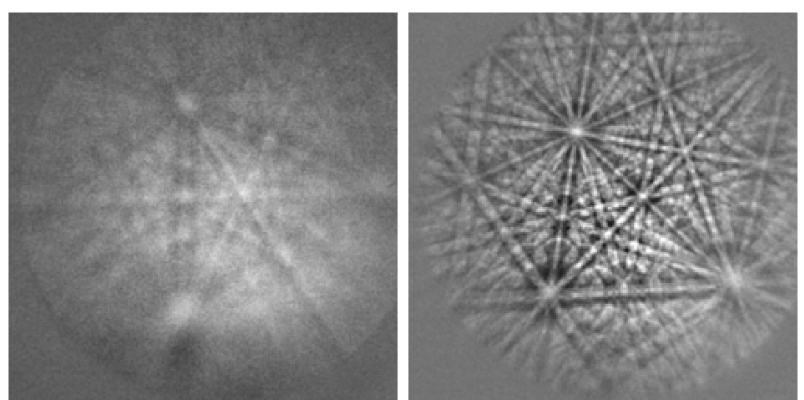

Figure 1. Diamond finish (left) vs. colloidal silica finish (right).
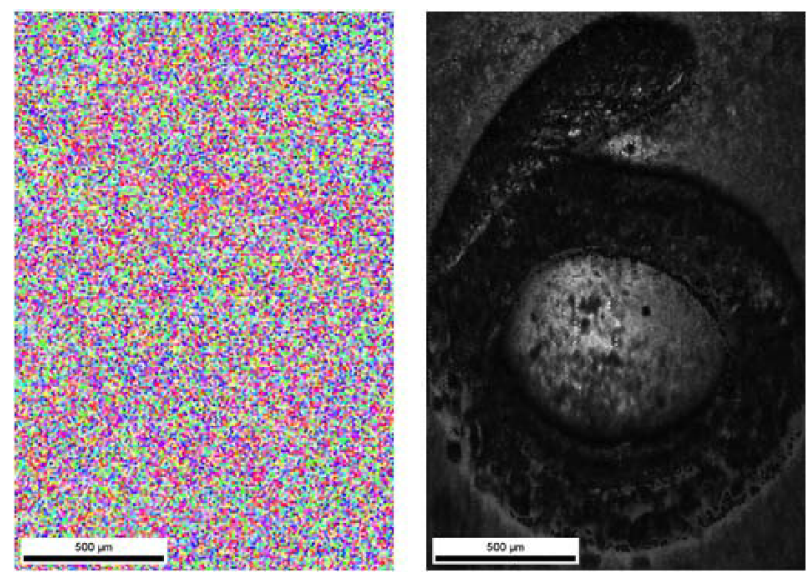

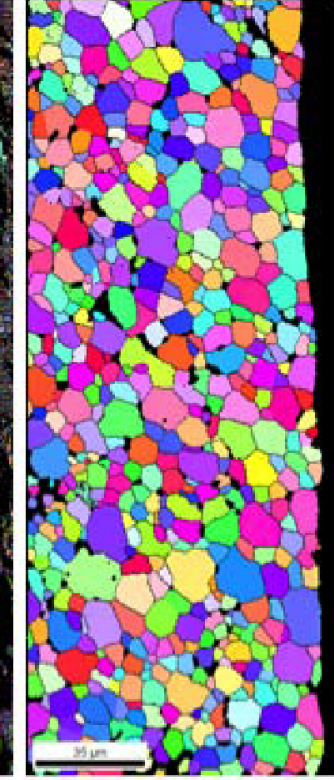

Figure 2. Small artificial grains (left) and properly partitioned structure showing voids (right).

Figure 3. Inverse pole figure map (left) and image quality map (right). 